\title{
Unraveling Hydrophobic Interactions at the Molecular Scale Using Force Spectroscopy and Molecular Dynamics Simulations
}

\author{
Philipp Stock, ${ }^{\dagger}, \|$ Jacob I. Monroe, ${ }^{\ddagger}, \|$ Thomas Utzig, ${ }^{\dagger}$ David J. Smith, ${ }^{\dagger}$ M. Scott Shell, ${ }^{, \dagger}$
} and Markus Valtiner* ${ }^{*},, \S$

${ }^{\dagger}$ Department for Interface Chemistry and Surface Engineering, Max-Planck-Institut für Eisenforschung GmbH, D-40237 Düsseldorf, Germany

${ }^{*}$ Department of Chemical Engineering, University of California Santa Barbara, Santa Barbara, California 93106-5080, United States

${ }^{\S}$ Department for Physical Chemistry, Technische Universität Bergakademie Freiberg, D-09599 Freiberg, Germany

\section{Supporting Information}

ABSTRACT: Interactions between hydrophobic moieties steer ubiquitous processes in aqueous media, including the self-organization of biologic matter. Recent decades have seen tremendous progress in understanding these for macroscopic hydrophobic interfaces. Yet, it is still a challenge to experimentally measure hydrophobic interactions (HIs) at the single-molecule scale and thus to compare with theory. Here, we present a combined experimental-simulation approach to directly measure and quantify the sequence dependence and additivity of HIs in peptide systems at the single-molecule scale. We combine dynamic single-molecule force spectroscopy on model peptides with fully atomistic, both equilibrium and nonequilibrium, molecular dynamics (MD) simulations of the same systems. Specifically, we mutate a flexible (GS) 5 peptide scaffold with increasing numbers of hydrophobic leucine monomers and measure the peptides' desorption from hydrophobic self-assembled monolayer surfaces. Based on the analysis of nonequilibrium work-trajectories, we measure an interaction free energy that scales linearly with 3.0-3.4 $k_{\mathrm{B}} T$ per leucine. In good agreement, simulations indicate a similar trend with $2.1 k_{\mathrm{B}} T$ per leucine, while also providing a detailed molecular view into HIs. This approach potentially provides a roadmap for directly extracting qualitative and quantitative single-molecule interactions at solid/liquid interfaces in a wide range of fields, including interactions at biointerfaces and adhesive interactions in industrial applications.

KEYWORDS: single-molecule force spectroscopy, AFM, molecular dynamics, steered molecular dynamics, Jarzynski's equality, hydrophobic interaction, peptide, self-assembled monolayer

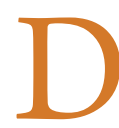
espite over 30 years of extensive research, discerning the origin, scaling, and even existence of forces between extended hydrophobic surfaces remains a major experimental challenge. Direct measurements of the hydrophobic interaction (HI) not relying on inference from macroscopic properties have been notoriously difficult to execute without interposing physical forces and processes, such as bridging bubbles and surfactant overturning. In their recent review, Ducker and Mastropietro ${ }^{1}$ identify only five experimental results at extended hydrophobic interfaces that show convincing evidence for exponentially decaying attractive HIs in the range of $3-12 \AA{ }^{2-6}$ where reexamination of the large body of remaining literature reveals principal experimental difficulties. ${ }^{1}$ Here, we use 'hydrophobic interaction' to refer to the total interaction free energy between solvated hydrophobes and not specifically the water-mediated contribution. In contrast at small length scales, nonlinear optical spectroscopy studies ${ }^{7,8}$ and diffraction experiments ${ }^{9}$ have shown how water orients and fluctuates around uncharged small hydrophobes, which may be critical to molecular HI forces, even if such forces themselves have been difficult to measure. Indeed, molecular theory and simulations continue to clarify the relationship between fluctuating water structure and the hydrophobicity of isolated solutes and interfaces. ${ }^{10,11}$

Understanding how $\mathrm{HI}$ forces manifest in a molecular context and can be systematically manipulated, e.g., through variations in amino acid sequence, remains a major unsolved

Received: September 20, 2016

Accepted: March 7, 2017

Published: March 7, 2017 
problem. This is particularly relevant to peptides, which depart from the idealized solutes often studied in theory as they comprise nonrigid, heterogeneous molecules with both hydrophobic and hydrophilic groups. For realistic sequences containing a variety of amino acids, a delicate balance of noncovalent driving forces, including the $\mathrm{HI}$ as a significant contributor, directs peptide aggregation, self-assembly, folding, and binding behaviors. ${ }^{12-14}$ Several recent single-molecule spectroscopic studies have now probed polylysine, ${ }^{{ }_{5}}$ spider silk peptides, ${ }^{16}$ or charged/hydrophobic peptide sequences ${ }^{17}$ with extended hydrophobic surfaces, ${ }^{18-20}$ but systematically understanding how the HI scales with the hydrophobic content in a peptide sequence has yet to be clarified.

Here, we examine the scaling of interactions between a short peptide of mixed hydrophilic/hydrophobic character with an extended hydrophobic surface as the peptide hydrophobic content is adjusted. Related studies on the single-molecule level have focused on homopolymers ${ }^{21}$ or homopeptides. ${ }^{19}$ By mutating a hydrophilic scaffold to increase the number of hydrophobic residues, we can instead probe the additivity and potential cooperation or competition between hydrophilic and hydrophobic moieties related to surface affinity. Specifically, we combine direct experimental measurements using singlemolecule AFM (SM-AFM) with fully atomistic nonequilibrium and equilibrium molecular dynamics (MD) simulations of the same systems, finding good agreement without any systemspecific fitted parameters. In both experiment and simulation, we assess the strength of the peptide-surface interaction by rigorously applying Jarzynski's equality (JE) to determine equilibrium free energy differences. Finally, we show that these equilibrium free energy differences may be associated with changes in the equilibrium ensemble of water structure at the level of three-body angular correlations.

\section{RESULTS AND DISCUSSIONS}

A comparison of the SM-AFM and MD experimental setups is shown in Figure 1. In experiment, fully designed peptide sequences with varying degrees of hydrophobicity are endgrafted with their N-terminus to a polyethylene glycol (PEG) linker, with 12 PEG units and a terminal $\mathrm{COOH}$ group $\left(\mathrm{PEG}_{12}-\mathrm{COOH}\right)$, on ultrasmooth gold surfaces modified with self-assembled monolayers (SAMs, $\sigma \approx 3 \AA$ ). ${ }^{5,22}$ We use a hydrophilic $(\mathrm{GS})_{5}$ model backbone of alternating glycine $(\mathrm{G})$ and serine $(S)$ amino acids, and the C-terminal amino acids are selectively mutated with up to four consecutively or nonconsecutively distributed hydrophobic leucine (L) units within the sequence (Table 1 ). With the backbone, the entire protein anchor has an estimated contour length, $L_{C}$, of $10 \mathrm{~nm}$. The surface chemistry is confirmed by photoelectron spectroscopy (XPS, see Figure S10 in the Supporting Information, SI). The grafted peptide mutations on the $\mathrm{PEG}_{12}$-linker are brought into contact with a 1-undecanethiol terminated hydrophobic goldcoated AFM tip, and single-molecule experiment force versus distance characteristics are recorded in so-called fly fishing mode. $^{23}$ In this experimental AFM technique, a tip contacts a surface at random points and if-in our context-a single grafted peptide adsorbs at the hydrophobic tip during contact, the formed hydrophobic contact breaks during separation of the tip and the surface, leading to a specific signature in the force profile. $^{23}$

The atomically detailed simulated system (shown in Figure 1B) is designed to closely approximate both the physical system and the experimental protocols. The $\mathrm{N}$-terminus of each of the
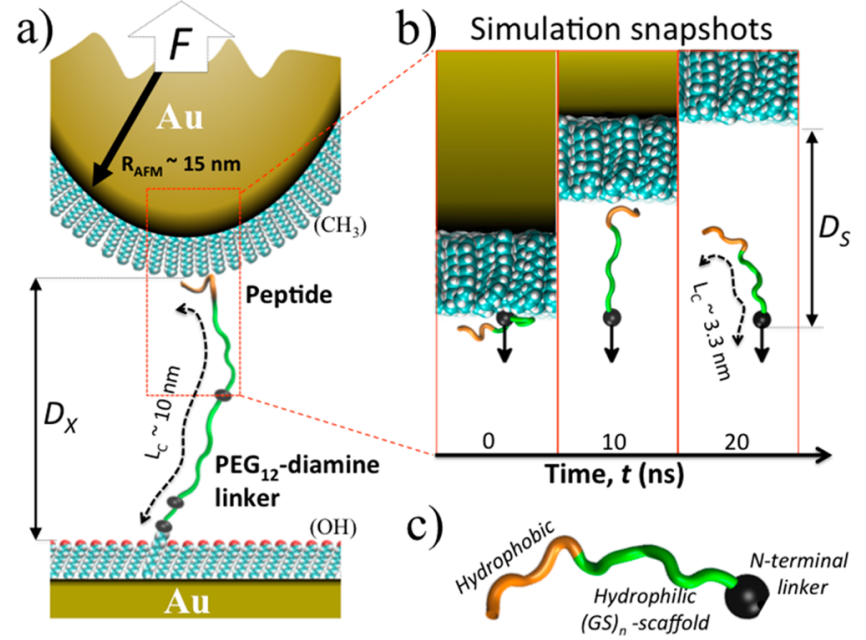

Figure 1. Schematic of (a) experimental and (b) computational setting used for quantifying dynamic HIs between a set of different hydrophobic peptides (shown in Table 1) and an extended hydrophobic surface as a function of the experimental distance $D_{\mathrm{X}}$ and the simulation distance $D_{\mathrm{S}}$, respectively. (c) Hydrophobic mutations are introduced specifically at the C-terminal tail end of a hydrophilic (GS) $)_{n}$-based scaffold. In experiment (a) a PEG PE- $^{-}$ diamine-based polymer linker is used to covalently graft peptides on a molecularly smooth $\mathrm{OH} / \mathrm{COOH}$ terminated SAM on a gold surface (roughness, $\sigma_{\mathrm{RMS}} \leq 2-3 \AA$ ). The contour length of $L_{\mathrm{C}}=10$ $\mathrm{nm}$ is indicated. Gold-coated AFM tips (radius, $R_{\mathrm{AFM}} \sim 15 \mathrm{~nm}$ ) are hydrophobized with a SAM and interactions between different surface grafted peptide mutations (see Table 1) and the hydrophobic tip are measured. In the simulation, the PEG linker is not included, and the contour length of the individual peptides amounts to $L_{\mathrm{C}} \sim 3.4 \mathrm{~nm}$. In (b) the $\mathrm{N}$-terminal peptide end and applied pulling direction are marked with an arrow.

peptides, which in experiment is tethered to a PEG linker and ultimately a hydrophilic surface, is pulled at constant velocity away from a fixed, periodically replicated hydrophobic SAM surface approximating the AFM tip. Numerous models of SAM surfaces, ${ }^{24-28}$ as well as SAM-peptide systems, ${ }^{29-35}$ have been proposed in the literature; here we build the SAM from dodecane molecules with a single end fixed at the necessary lattice spacing, with surrounding water explicitly represented.

Additional methodological details are described in the Materials and Methods. A complete set of results for all peptides used and additional data analyses, including those relevant to experiment and simulations, are available in the SI.

Experimental results in Figure 2A show a master curve with $\sim 80$ individual trajectories recorded during unbinding of the (GS) ${ }_{3} \mathrm{~L}_{4}$-sequence (see Table 1 ) from a hydrophobic AFM tip. As described previously, all shown trajectories are aligned by pulling distance and time using the best worm-like-chain (WLC) fit. $^{36}$ The inset in Figure $2 \mathrm{~A}$ shows a typical individual force versus distance curve, with the distance normalized by the contour length, $L_{\mathrm{C}}$, of the molecular anchor. About 3-8\% of force profiles indicate successful surface-to-molecule binding and hence allow direct dynamic measurement of worktrajectories during unbinding of single-molecule HIs. For all other peptide sequences, similar master curves recorded at different loading rates are shown in Figure S12. Curves without successful bond formation show only van der Waals (vdW), hydration, and electric double-layer interactions that can be fit well with an extended DLVO theory. ${ }^{37,38}$ Control experiments 
Table 1. Peptide Mutation Sequences and Summary of Experimentally Obtained and Simulated Interaction Free Energies with Extended Hydrophobic Surfaces

\begin{tabular}{|c|c|c|c|c|c|}
\hline $\begin{array}{l}\text { mutation } \\
\text { sequence }\end{array}$ & $\begin{array}{l}\text { experiment } \Delta G \\
\left(k_{\mathrm{B}} T\right)\end{array}$ & $\begin{array}{l}\text { simulation fast-pull } \Delta G \\
\qquad\left(k_{\mathrm{B}} T\right)\end{array}$ & $\begin{array}{l}\text { simulation slow-pull } \Delta G \\
\qquad\left(k_{\mathrm{B}} T\right)\end{array}$ & $\begin{array}{l}\text { simulation equilibrium umbrella } \\
\text { sampling } \Delta G\left(k_{\mathrm{B}} T\right)\end{array}$ & $\begin{array}{l}\text { three-body angle model } \Delta G \\
\qquad\left(k_{\mathrm{B}} T\right)^{a}\end{array}$ \\
\hline$-\mathrm{N}(\mathrm{GS})_{5}$ & $\begin{array}{c}\text { below detection } \\
\text { limit }\end{array}$ & $20.9 \pm 1.7$ & $7.4 \pm 0.5$ & $4.6 \pm 0.1$ & N/A \\
\hline$-\mathrm{N}(\mathrm{GS})_{4} \mathrm{GL}$ & $7.6 \pm 0.8$ & $17.5 \pm 3.2$ & $10.3 \pm 0.7$ & $8.0 \pm 0.1$ & 7.8 \\
\hline$-\mathrm{N}(\mathrm{GS})_{4} \mathrm{~L}_{2}$ & $8.8 \pm 0.9$ & $22.9 \pm 4.6$ & $13.4 \pm 2.4$ & $7.9 \pm 0.1$ & 10.5 \\
\hline$-\mathrm{N}(\mathrm{GS})_{3} \mathrm{GL}_{3}$ & $12.2 \pm 1.1$ & $31.1 \pm 2.4$ & $10.5 \pm 3.3$ & $11.6 \pm 0.1$ & 13.0 \\
\hline$-\mathrm{N}(\mathrm{GS})_{3} \mathrm{~L}_{4}$ & $17.9 \pm 0.8$ & $33.3 \pm 2.1$ & $16.7 \pm 1.5$ & $13.1 \pm 0.1$ & 14.6 \\
\hline$-\mathrm{N}(\mathrm{GS})_{3}(\mathrm{GL})_{2}$ & $9.6 \pm 0.6$ & - & - & - & - \\
\hline$-\mathrm{N}(\mathrm{GS})_{2}(\mathrm{GL})_{3}$ & $14.0 \pm 0.7$ & - & - & - & - \\
\hline$-\mathrm{N}(\mathrm{GS})(\mathrm{GL})_{4}$ & $16.2 \pm 1.1$ & - & - & - & - \\
\hline
\end{tabular}

${ }^{a}$ Errors are not provided for $\Delta G$ values from the three-body angle model, since this is already an approximate theory utilizing reference probability distributions for which the uncertainties are difficult to rigorously assess.
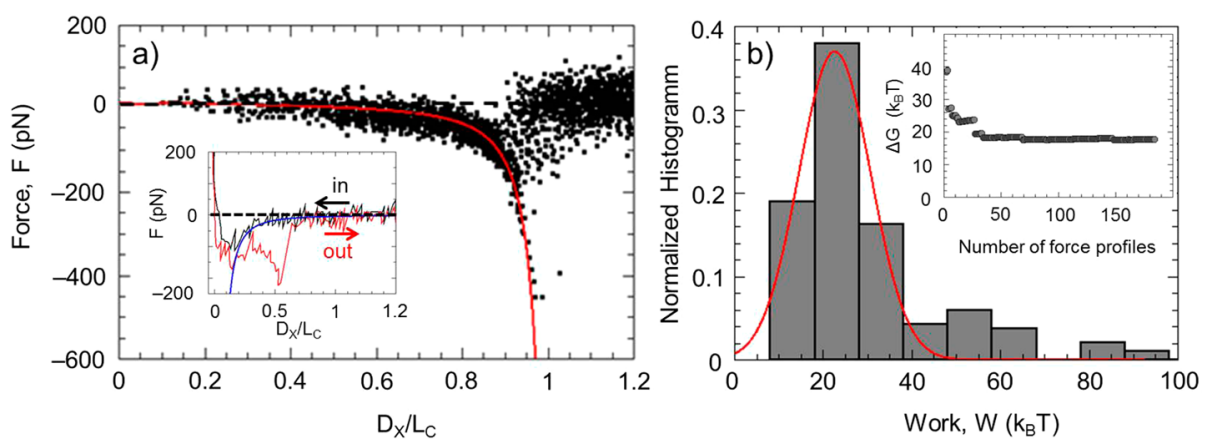

Figure 2. (a) Typical master curve with about 80 individual rupture events measured between -N-(GS) ${ }_{3}$ LLLL functionalized surface and hydrophobic tip, aligned by the best WLC fit with a contour length of $10 \mathrm{~nm}$ and a persistence length of $0.37 \mathrm{~nm}$. Inset shows a typical individual SM-AFM force distance profile with both a primary adhesive minimum and a single molecular rupture signature at $60 \%$ full extension of the linker. The inset shows a fit by an extended DLVO theory (for fit details see SI). (b) Normalized histogram of the measured work distribution. The inset in (b) shows Jarzynski's free energy $\Delta G$ as a function of the number of force trajectories.

using surfaces without grafted peptides are shown in Figure S11 as well.

On the basis of the data sets of individual pulling trajectories, the interaction free energy difference between the bound and unbound states of the different pulled peptides with the hydrophobic tip surface can be estimated using JE: ${ }^{39}$

$$
\mathrm{e}^{-\Delta G / k_{\mathrm{B}} T}=\left\langle\mathrm{e}^{-W / k_{\mathrm{B}} T}\right\rangle
$$

The free energy difference, $\Delta G$, is related to an exponential average of the mechanical work $W$ over an infinite number of realizations of the same nonequilibrium protocol that probe the full work distribution. In practice, an estimate for the free energy difference is given by the same equation using a finite number of realizations $n$ and work values $W_{i}(i=1,2, \ldots, n)$ calculated from individual pulling curves, ${ }^{36,40}$ such that the true difference is approached as $n \rightarrow \infty$.

Through Jarzynski averaging, one can hence exactly obtain the equilibrium free energy difference between the beginning and end states of a nonequilibrium process. A definition of these states is important for identifying and assessing the dissipated work in an experimental setting. In our experiments, the beginning state consists of a relaxed linker and peptide interacting with an AFM tip in equilibrium, while the end state corresponds to both the linker and peptide similarly relaxed (i.e., under no tension) but with the tip far away from the peptide. Thus, the equilibrium free energy difference between these two equilibrium states consists nearly totally of the contribution from the hydrophobic peptide-AFM tip interaction. We note that the macroscopic AFM spring is in zero position before and after the integration starts. As a consequence, and as shown in our previous work, integrating the measureable work from $t=0$, where the force $F=0$ until postbond rupture (where $F=0$ again), provides an excellent estimate of surface/molecule ${ }^{36}$ and intermolecular binding free energies. $^{41}$

In practice, the measured work includes a contribution to extend the PEG tether prior to bond rupture, after which point the tether relaxes back to equilibrium and its stored energy is dissipated. Thus, the linker affects the distribution of work values obtained, the extent of deviations from equilibrium behavior, and in turn how easily the Jarzynski average can be converged with a finite number of runs. However, in principle for an infinite number of runs, the calculated underlying free energy difference should be insensitive to the linker since it is in a relaxed configuration in both the beginning and end states. Long linkers will require more measurements of the process in order to converge the free energy difference, but again are not expected to significantly alter it. Moreover, in our case the short linker likely adopts a quasi-equilibrium state during most of the pulling process prior to rupture, as unfolding/refolding of a PEG linker (here $L_{\mathrm{C}} \sim 10 \mathrm{~nm}$ ) is very fast relative to the present AFM pulling rates. ${ }^{42,43}$ This behavior should further reduce spread introduced by the linker in the work distribution and, in turn, the finite-run sample bias in the Jarzynski free energy estimate. Information related to the convergence of our free energy estimates is provided in the SI, while error estimates and bias are discussed in more detail below. We also note that Hummer and Szabo discuss in great detail the physics of 
extracting specific free energy contributions from nonequilibrium measurements and their evolution along a pulling coordinate that involves linker stretching measured by macroscopic spring devices. ${ }^{44,45}$

Using JE we can hence estimate the interaction free energies for all designed peptide permutations interacting with the hydrophobic tip at three different loading rates (see Table S4 and Figure S12). ${ }^{36,40}$ In Figure 3 the estimated JE interaction

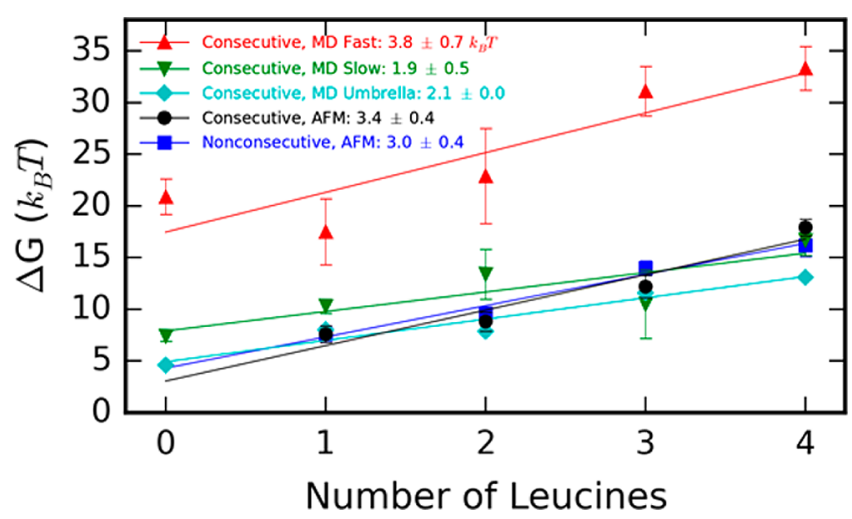

Figure 3. Comparison of experimentally and theoretically estimated interaction free energies as a function of the number of leucines. For the fast-pulling $(10 \AA / \mathrm{ns})$ and slow-pulling $(2 \AA / \mathrm{ns})$ MD cases, the $R^{2}$ values for the linear fits are 0.71 and 0.80 , respectively. All other $R^{2}$ values are $>0.90$.

free energies are shown for all peptides. Interestingly, the overall interaction scales approximately linearly with the number of leucine units, with fits showing that each additional hydrophobic unit contributes $3.4 \pm 0.4 k_{\mathrm{B}} T$ for consecutive and $3.0 \pm 0.4 k_{\mathrm{B}} T$ for nonconsecutive leucine mutations (see again Table 1). This falls within the range of values measured by macroscopic bulk methods for various interacting hydrophobes $^{46}$ and is consistent with simulation dimerization free energies for methane $\left(\sim 1.2-3.4 k_{\mathrm{B}} T\right),{ }^{47,48}$ cyclohexane (4.6 $\left.k_{\mathrm{B}} T\right){ }^{46}$ and benzene $\left(3.4 k_{\mathrm{B}} T\right){ }^{49}$

More interestingly, the HI free energy of 3.0-3.4 $k_{\mathrm{B}} T$ per leucine unit describes all mutation results within experimental uncertainty, whether hydrophobic units are located next to each other or separated by a glycine. This suggests that the mutations used are intrinsically unstructured and interact noncooperatively with the hydrophobic surface. MD simulations of the peptides in bulk and at the surface support this interpretation, showing that none of the mutations have pronounced formation of secondary structures (Figures S2S4). In solution, all simulated peptides exhibit similar and somewhat broad distributions for radius of gyration $\left(R_{\mathrm{g}}\right)$, solvent-accessible surface area (SASA), and number of intrapeptide hydrogen bonds that is suggestive of a diverse structural ensemble. The same distributions do reveal some sequence-specific signatures when the peptides are near the hydrophobic surface, but they remain broad and are consistent with unstructured behavior.

These observations strongly suggest a linear model for the peptide-surface interaction free energy with the number of leucines present:

$$
\Delta G=n_{\mathrm{L}}\left(\Delta g_{\mathrm{L}}-\Delta g_{\mathrm{GS}}\right)+n_{\mathrm{tot}} \Delta g_{\mathrm{GS}}
$$

Here, $n_{\mathrm{L}}$ is the number of leucines, $n_{\text {tot }}$ is the number of residues excluding caps, and $\Delta g_{\mathrm{L}}$ and $\Delta g_{\mathrm{GS}}$ are the free energies of removing a single leucine or (averaged) glycine/serine residue from the surface, respectively. Thus, in Figure 3, the intercept is given by the interaction free energy of the scaffold with the surface, and the slope by the difference in interaction free energy of a leucine residue versus that of the scaffold (Table 2). Similar additivity for the solvation of small hydrophobic monomer units in a homopolymer was previously noted by $\mathrm{Li}$ and Walker. ${ }^{50}$ Within experimental uncertainty and in the context of this model, the mutation of a peptide residue from hydrophilic to hydrophobic has an additive effect on its interaction with a hydrophobic surface, regardless of the proximity (spaced or adjacent) to other hydrophobic monomers. Thus, we do not detect any cooperativity or anticooperativity between the hydrophobic units, although it is possible that such effects may be present at a level smaller than the experimental uncertainty.

Simulation-calculated free energies of peptide removal follow the same approximately linear trend as in experiments, but are slightly higher than those determined experimentally and increase with pulling rate (Table 1). This is not surprising for at least two reasons: simulated pulling rates are necessarily orders of magnitude faster than the $\sim 10^{-4} \AA / \mathrm{ns}$ of experiments, and the simulations are also limited to fewer pulling runs in each case (60 at the slowest rate). Both factors magnify the statistical bias introduced by JE. It is evident from eq 1 that this bias originates in the asymmetric weighting by the exponential of low work values in the averaging procedure; thorough sampling of rare trajectories, with work values well below the mean, is thus necessary for convergence. When the number of runs is limited, as here, our calculations (Table S1) show that a single pulling curve with a low work value skews the free energy calculations.

It is well-known that the bias introduced by JE for finite sample sizes is nontrivial ${ }^{51}$ and increases with pulling rate and nonequilibrium driving; therefore, we suspect that both the experimental and simulated slopes are overestimated to some degree. In both, the bias appears more significant for faster pulling, and in simulation it also increases with the number of leucines, as illustrated by the decrease in slope moving from the fast to slow rate in Figure 3. Section 1 (SI) describes a procedure to approximate it for each peptide using these results. In the experiments, we estimate a nonsystematic bias

Table 2. Fitting Parameters for Free Energy Model Linear in the Number of Leucines ${ }^{a}$

\begin{tabular}{|c|c|c|c|c|c|}
\hline$\left(k_{\mathrm{B}} T\right)$ & experiment nonconsecutive & experiment consecutive & simulation fast-pull & simulation slow-pull & simulation equilibrium \\
\hline slope & $3.0 \pm 0.4$ & $3.4 \pm 0.4$ & $3.8 \pm 0.7$ & $1.9 \pm 0.5$ & $2.1 \pm 0.0$ \\
\hline intercept & $4.3 \pm 1.0$ & $3.1 \pm 1.0$ & $17.5 \pm 1.9$ & $7.9 \pm 0.7$ & $4.9 \pm 0.1$ \\
\hline$\Delta g_{\mathrm{GS}}$ & $0.4 \pm 0.1$ & $0.3 \pm 0.1$ & $1.7 \pm 0.2$ & $0.8 \pm 0.1$ & $0.5 \pm 0.0$ \\
\hline$\Delta g_{\mathrm{L}}$ & $3.5 \pm 0.4$ & $3.7 \pm 0.4$ & $5.6 \pm 0.7$ & $2.7 \pm 0.5$ & $2.6 \pm 0.0$ \\
\hline
\end{tabular}

${ }^{a} \Delta g_{\mathrm{GS}}$ is the free energy change per residue of removing the scaffold from the surface, and $\Delta g_{\mathrm{L}}$ is the free energy change of removing a single leucine residue. 
between $2 \pm 1 k_{\mathrm{B}} T$ for the slowest experimentally recorded pulling rates of $0.5 \mu \mathrm{m} / \mathrm{s}$. The bias increases to $4-6 k_{\mathrm{B}} T$ for 1.5 $\mu \mathrm{m} / \mathrm{s}$ and up to $8-10 k_{\mathrm{B}} T$ for the fastest experimentally applied pulling rate of $2 \mu \mathrm{m} / \mathrm{s}$, while rare low-work events still result in almost no variation of the estimated free energy, irrespective of the pulling rate (Table S3). In all of these cases, the bias does not seem to affect the slope in Figure 3, such that the experimental HI per leucine appears well approximated in the quoted range from 3 to $3.4 k_{\mathrm{B}} T$.

The use of both forward and reverse (toward the surface) pulling simulations with bidirectional free energy estimators, such as Bennet's acceptance ratio (BAR), has also been shown to reduce systematic bias in nonequilibrium studies like this. ${ }^{52}$ The reverse protocol is not possible in experiment for the present scenario, but we do compute these with simulations. Still, we find that convergence of simulation reverse work distributions is poor (Tables $S 1-S 2$ ). Here the BAR formula is also sensitive to rare, negative reverse work values from trajectories in which the pulling restraint actively fights against the motion of the peptide as it is attracted toward the surface. Close inspection of Figure 4A reveals that these rare trajectories are not well-sampled across all peptides due to the stochastic nature of the simulations (see SI for additional discussion).

To better understand the limitations of the nonequilibrium pulling measurements and JE, we perform comparative equilibrium simulations to calculate free energy differences. Specifically, we use the umbrella sampling approach in which the peptide-surface $z$-distance fluctuations are restrained to a small window using a harmonic potential. As described in Materials and Methods, thermodynamic reweighting techniques then link together multiple such simulations, with systematically varying windows, to produce detailed free energy curves and differences. Free energy differences are computed between a potential of mean force (PMF) curve's minimum and asymptote at far distances. Interaction free energies computed from these equilibrium simulations are generally smaller than those from JE (Table 1), as expected, yet yield a slope that is in good agreement with slower pulling simulation runs at $2.1 k_{\mathrm{B}} T$ per leucine.

Finally, we note that the hydrophilic $(\mathrm{GS})_{5}$ scaffold is not included in determining the free energy per leucine for experiments because we are not able to measure its interactions with the surface (see Figure S11). Table 1 shows that the scaffold has a $\sim 3 k_{\mathrm{B}} T$ smaller interaction free energy than the (GS) ${ }_{4} \mathrm{GL}$ peptide when calculated from simulations. Interestingly, the simulation runs at faster pulling rates predict the scaffold affinity to be large, which is an artifact due to a biasing of the configuration of $(\mathrm{GS})_{5}$ near the surface due to the applied restraint (Figure S2); the bias is less significant at slower pulling rates that allow the peptide to well-equilibrate with the surface. It should also be re-emphasized that, while the presence of a surface limits the conformational space explored by the peptides compared to fully solvated configurations, all lack well-defined structures in both states (Figures S2-S4). Moreover, all peptides have relatively fast conformational rearrangement times (assessed by the $R_{\mathrm{g}}$ autocorrelation time) at $0.5-2.0 \mathrm{~ns}$ on the surface and slightly longer in solution (Figure S5).

In experiment, we are limited to analyzing interaction free energies and measured forces, while the nonequilibrium simulations, which nearly quantitatively reproduce these experimental results, suggest a molecular, mechanistic picture of the pull-off process and in turn various contributions to the
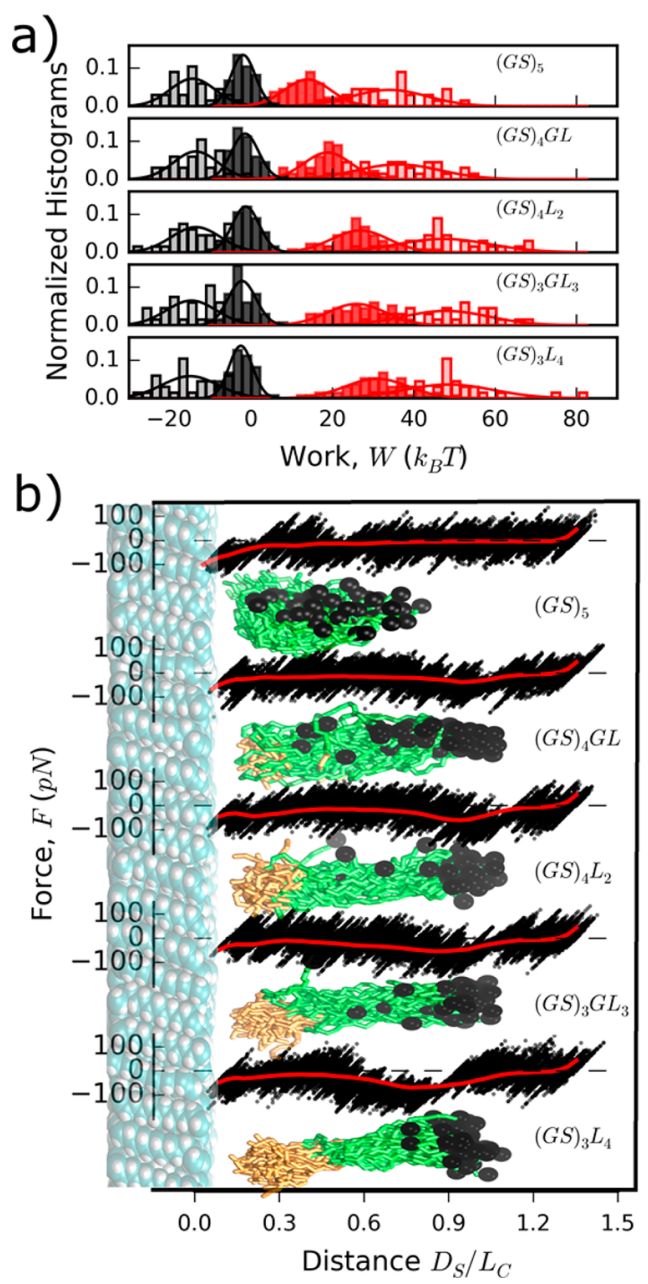

Figure 4. (a) Forward, $P\left(W_{\text {for }}\right)$ (red), and negated reverse, $P\left(-W_{\text {rev }}\right)$ (black), work distributions show that slower pulling rates ( $2 \AA / \mathrm{ns}$, solid bars) significantly reduce the hysteresis and spread observed at faster pulling speeds (10 ̊//ns, lightly shaded). At slower pulling rates, many negative work values (positive here, as negated reverse distributions are shown) are observed during reverse pulling runs. (b) Black dots are force versus distance profiles from single, typical trajectories, and red lines are force profiles averaged over all trajectories. Inset structures show peptide chains in green (hydrophilic) and orange (hydrophobic) and pulled atoms (black) at the time of pull-off for all trajectories of a particular peptide. These structures are sized and aligned such that they are proportional to the scale on the $x$-axis.

work distributions. For example, Figure 4B shows that detachment for $(\mathrm{GS})_{5}$ is quite different from that of the other peptides; once even a single leucine is introduced, the peptide extends substantially prior to detachment in order to maintain leucine residues near the surface (also see Video S1 of the SI for the $(\mathrm{GS})_{3} \mathrm{~L}_{4}$ case). This trend is manifested in peptide pull-off time and distance, radius of gyration, numbers of shell waters, and SASA (Figures S6-S7).

Simulations also show different regimes of the workdistance curves associated with distinct underlying molecular mechanistic processes (Figure 5): (1) initial perturbation from a stable surface configuration; (2) low-tension peptide extension; (3) high-tension extension in which the peptide exhibits WLC behavior; and (4) dragging through solution. The work for the $(\mathrm{GS})_{5}$ peptide primarily lies in the first regime, but as leucines are added, contributions from the high- 

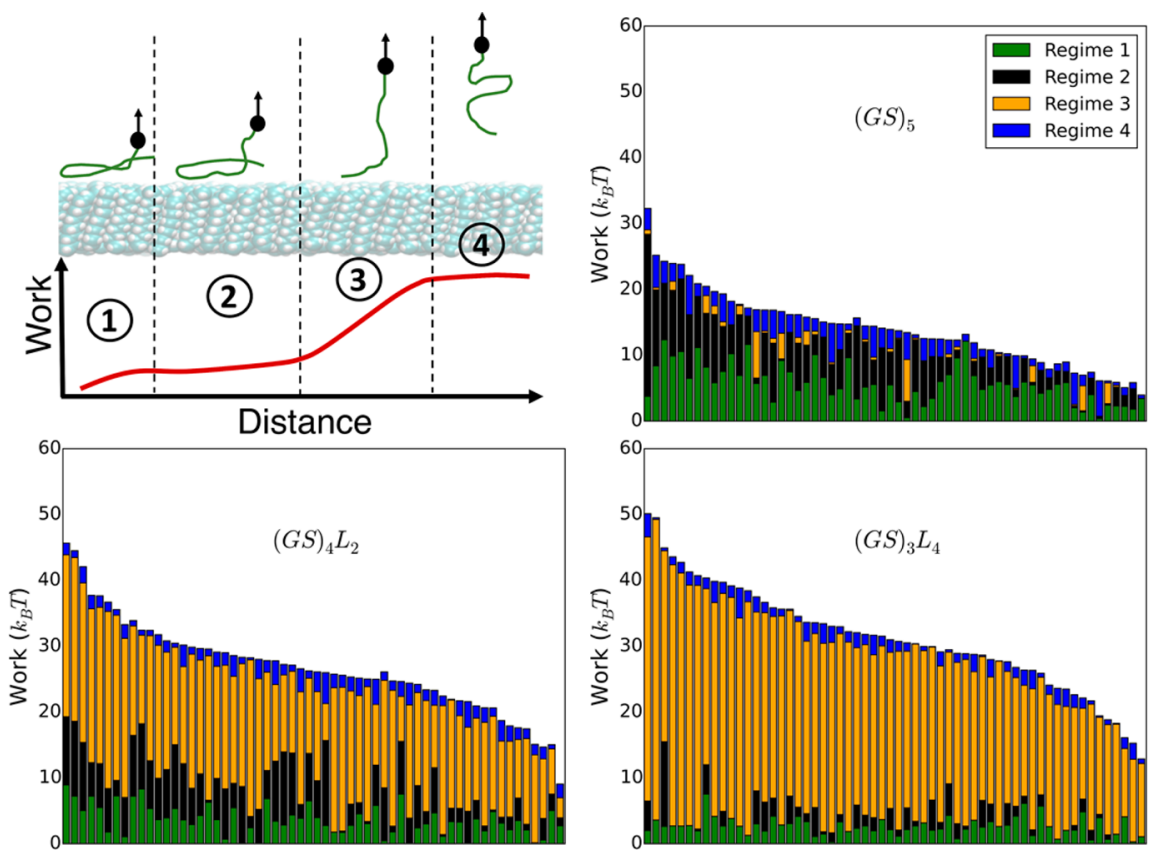

Figure 5. Contributions to the work are shown from each pulling regime for slow-pulling runs of the simulated peptides. Pulling regimes correspond to changes in the slope of the work versus distance curve and are physically defined as: (1) from the beginning of the simulation until the pulled atom is off the surface; (2) until the centers of geometry of one less than the number of nonleucine residues are off the surface; (3) until the last atom in the peptide is off the surface; and (4) from pull-off until the end of the simulation. For all events described, the cutoff for being on the surface is set to a height of $21 \AA$ above the fixed carbons of the dodecane molecules. Without any leucines, the largest contribution to the work appears in regime 1 or the perturbation of the peptide's configuration on the surface. With addition of leucines, stronger interactions result in larger contributions to the work after the peptide is extended (regime 3).

extension regime grow, reflecting the increased strength of the peptide-surface interaction. For all leucine-containing peptides, work contributions for surface perturbation and dragging through solution are roughly equivalent. Interestingly, this matches the expectation that HIs, and thus surface affinity, should grow with the number of leucine residues present, with the largest energy barrier to detachment being the creation of hydrophobic surface areas.

Importantly, the simulations reveal molecular-structural factors associated with the linear increase in binding free energy with the number of leucines. For example, the bare scaffold shows an increase in peptide-water and a decrease in intrapeptide hydrogen bonds upon its removal from the surface, but the trend gradually shifts as leucines are added until it is inverted for the $(\mathrm{GS})_{3} \mathrm{~L}_{4}$ case (Figure 6). Moreover, the additional hydrophobic surface area exposed upon detachment from surface into solution generally increases with the number of leucines, and such that each leucine independently contributes a relatively equal amount to the total change in hydrophobic surface area. As more are added, however, the peptide backbone geometry does not permit all leucines to directly contact the surface, forcing at least one leucine in the (GS) ${ }_{3} \mathrm{GL}_{3}$ and $(\mathrm{GS})_{3} \mathrm{~L}_{4}$ cases to remain partially or fully solvated when the peptide is near to the SAM (see also Figure S2 for visual renderings).

In such molecularly sized systems, it is not expected that the changes in interaction free energies should follow a macroscopic trend by scaling with the hydrophobic surface area. Indeed, we find that the effective surface tension, computed as the free energy change per change in hydrophobic SASA, varies with the number of leucines. Such an analysis neglects the contributions of the hydrophilic portion of the peptide, but more importantly ignores well-known differences in the nature

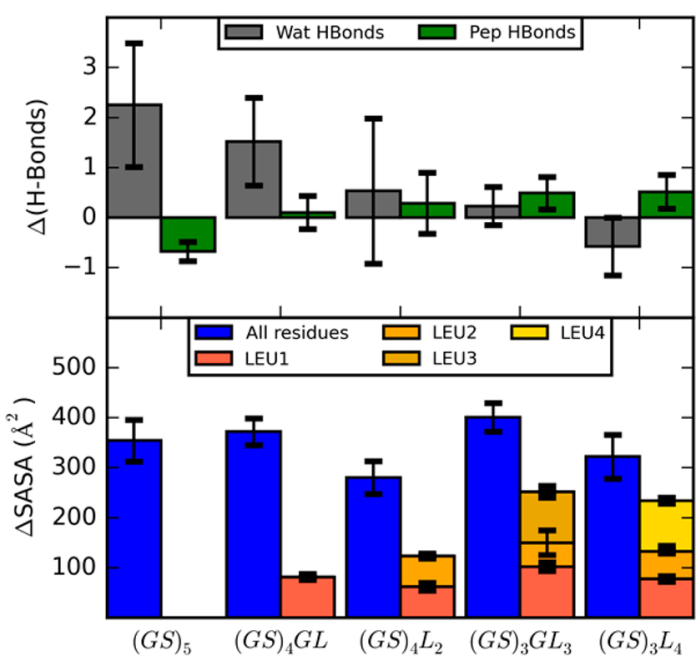

Figure 6. Change in average peptide properties moving from a surface-restrained state to solution. (a) With increasing leucines, peptides gain fewer peptide-water and more intramolecular hydrogen bonds in moving from the surface to solution, consistent with the slight increase in $\alpha$-helical tendency (Figure S2). (b) Changes in SASA are similar between peptides, while changes in hydrophobic SASA roughly increase with the number of leucines. Contributions to $\triangle \mathrm{SASA}_{\text {hyd }}$ are broken down by leucine: LEU1 refers to the leucine closest to the C-terminus of the peptide, LEU2 the next inward along the chain, and so on. Note that for $(G S)_{3} L_{4}$, backbone constraints prevent all four leucines from simultaneously becoming solvent shielded in the surface-associated state (Figure S2).

of the $\mathrm{HI}$ for small and large hydrophobes. ${ }^{53}$ Namely, hydration of small hydrophobes $(<1 \mathrm{~nm})$ tends to be entropically 
unfavorable due to water's unique cavity fluctuations and scales with cavity volume, whereas large ones $(>1 \mathrm{~nm})$ pay an enthalpic penalty for solvation that scales with exposed solute surface area. ${ }^{53}$ Recent efforts have also nuanced these ideas in terms of water-solute interaction energies, ${ }^{10}$ solute-local density fluctuations, ${ }^{54}$ and orientation of water dipoles. ${ }^{55}$ However, it is difficult to directly apply these kinds of analyses to the present peptide-surface systems due to the fluctuating, nonrigid nature of the peptide conformations and to the statistical accuracy with which the relevant quantities can be determined in these large, computationally expensive runs.

To seek molecular signatures that may delineate distinct regimes of $\mathrm{HI}$ physics, we thus characterize hydration water structure at the initial and final equilibrium states of the pulling process (Figure 7). Longstanding views have sought to

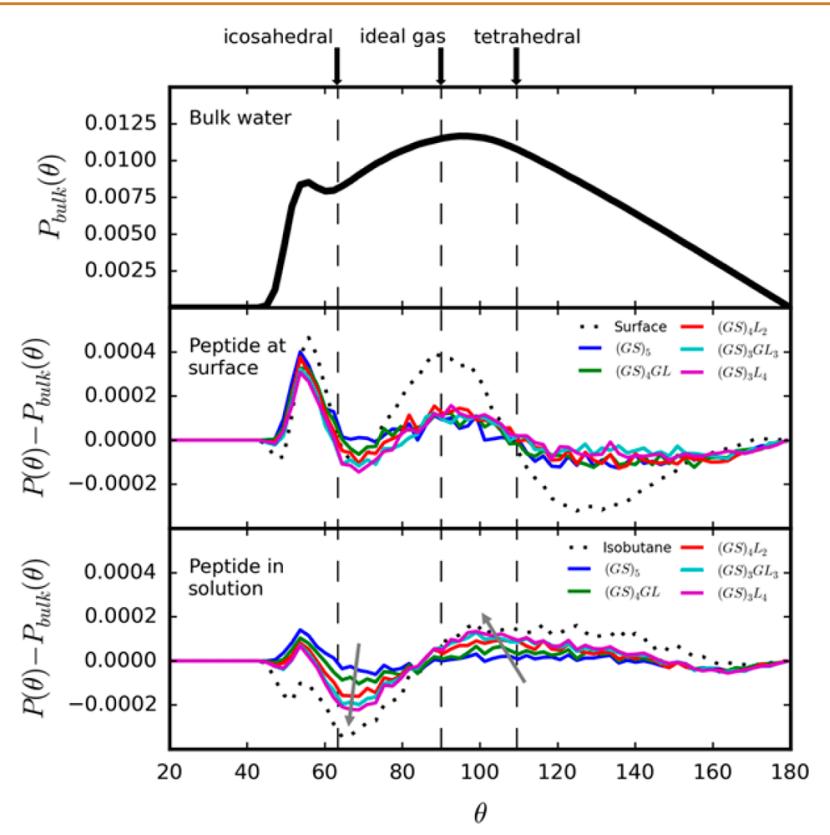

Figure 7. (Top panel) Probability density distribution of the triplet angle formed by water molecules with two nearest neighbors, in which the angle is measured at the oxygen site. For comparison, a simple Lennard-Jones fluid with icosahedral coordination has a distribution peaked slightly below $60^{\circ}$ with a much smaller secondary peak just above $109^{\circ}$. An ideal gas, non-interacting fluid has a broad distribution with a peak near $90^{\circ}$. (Middle and bottom panels) Changes to the triplet angle distribution for the peptide-SAM systems. The dashed curve in the central figure pertains to hydration waters within $5 \AA$ of the SAM surface, while the dashed curve at bottom refers to waters near a fully solvated isobutane molecule. Other curves give the perturbation to the system distribution for each peptide either restrained on the surface (middle) or far away in solution (bottom panel). Gray arrows show changes with increasing leucines, which, with the exception of the three and four leucine distribution comparison, are found to be statistically significant at the $5 \%$ confidence level by the Kolmogorov-Smirnov test.

understand HIs in terms of water's unique tetrahedral correlations, ${ }^{56-60}$ and to quantify these, we calculate distributions of the angle formed by triplets of coneighboring water molecules within a 5 A hydration shell of both the peptide and SAM surface. In bulk water this distribution peaks near the tetrahedral angle $\left(109.5^{\circ}\right)$, but surfaces and molecules can either induce enrichment or depletion of tetrahedral, random $\left(\sim 90^{\circ}\right.$, ideal gas-like $)$, or icosahedral $\left(\sim 60^{\circ}\right.$, simple liquid-like $)$ populations. ${ }^{56}$ As controls for comparison, we characterize how the extended hydrophobic SAM surface alone perturbs water structure (large-scale physics) versus how a single solvated isobutane, a leucine side-chain analogue, does the same (smallscale). We find that the bare SAM enriches the uncorrelated and icosahedral populations of waters, indicating a reduction in tetrahedral configurations, while the opposite is true for isobutane.

These reference cases provide context for the interpretation of similar distributions near interfacial and bulk-solvated peptides. For peptides on the surface, nearby waters exhibit behavior like those near the surface itself, with very little sequence-specific variation. In this context, it appears that water near the SAM surface (with or without peptide) exhibits weaker tetrahedral preference, as expected from large-scale hydrophobicity theory. ${ }^{53}$ Interestingly, however, when the peptides have been pulled off and into solution, the tetrahedral population grows and increasingly so with the number of leucines. While a cluster of one or two leucines should still act as a small hydrophobe in solution, three or four if grouped together should begin to enter the large-scale regime beyond 1 $\mathrm{nm}$. The surprising fact that the tetrahedral region of the angle distribution systematically increases with the number of leucines suggests that each is solvated in an independent fashion from the remaining, possibly due to geometric restrictions imposed by the backbone. Similar trends are also observed in the solvation free energies of moderately shortchain $n$-alkanes showing that collapse is not favorable, leaving the alkane in an extended conformation. ${ }^{61}$

Because the angle distributions of the peptides on the surface all look similar, one might imagine that the sequence variation in the bulk-solvated peptides (far from the surface) may signal the variation of association free energy alone. To examine the relation of shifts in three-body angle distributions to interaction free energies, we conduct equilibrium umbrella-sampling simulations for an isobutane molecule with the SAM surface, which gives a surface-to-bulk free energy change of $5.4 k_{\mathrm{B}} T$ (Figure S9). We define a per-water solvation penalty given by $5.4 k_{\mathrm{B}} T$ normalized by the average number of waters in the isobutane hydration shell. We hypothesize that the number of waters around a peptide that exhibit three-body angles similar to the isobutane distribution contribute this penalty to the overall association free energy. Similarly, we hypothesize that the remaining waters exhibit an angle distribution associated with the $(\mathrm{GS})_{5}$ scaffold and contribute based on a reference scaffold per-water solvation free energy.

To apply these ideas, we fit each peptide's three-body angle distribution as a weighted sum of the distributions for the $(\mathrm{GS})_{5}$ scaffold in solution and isobutane in solution, which bracket all perturbations of the bulk water distribution made by the hydrophobe-containing peptides (Figure 7). This gives $f_{\text {iso }}$, the fraction of the distribution that looks like the isobutane one versus that of the scaffold. Given the model assumptions, a simple model for the association free energy is

$$
\Delta G=N_{\mathrm{W}, \mathrm{pep}}\left(f_{\text {iso }} \frac{\Delta G_{\text {iso }}}{N_{\mathrm{W}, \text { iso }}}+\left(1-f_{\text {iso }}\right) \frac{\Delta G_{\mathrm{GS}}}{N_{\mathrm{W}, \mathrm{GS}}}\right)
$$

Here, $N_{\mathrm{w}, \mathrm{x}}$ is the number of shell waters around either $\mathrm{x}=$ the peptide, isobutane, or (GS $)_{5}$ scaffold, and $\Delta G_{\mathrm{x}}$ is the free energy of removing such a group from the surface. Remarkably, consideration of only water structure near each leucinecontaining peptide produces free energies via the above 
model that closely agree with the actual values (Tables 1 ), with a slope of $2.3 k_{\mathrm{B}}$ T/LEU, only slightly higher than $2.1 k_{\mathrm{B}}$ T/LEU as estimated from detailed free energy calculations. Though this model might be improved through more explicit consideration of how mutations affect peptide chain entropies, it seems clear that three-body structural information of water may be a powerful indicator of solute-solute and solvation thermodynamics.

In summary, these results highlight qualitatively distinct structural behavior for waters in the vicinity of the extended hydrophobic interface versus near the molecularly sized hydrophobic leucine side chains. Moreover, the three-body angle distribution for hydration waters around a peptide evolves with added leucines in a way that closely tracks the free energies, moving toward that of the purely hydrophobic isobutane side-chain analog. The angle distribution thus offers a convenient signature of $\mathrm{HI}$ regimes suitable to flexible, heterogeneous, soft-matter systems.

\section{CONCLUSIONS}

In summary, this combination of experimental and simulation approaches provides a molecular view into dynamic singlemolecule-surface interactions under applied load, in particular the all-important $\mathrm{HI}$ in the context of peptides, which may easily extend to arbitrary other surface-molecule interactions. The experimental and theoretical experiments are in good agreement given the precision of the measured data, with no parameters fit between the two. For the interaction of hydrophobic model scaffolds, we find that hydrophobic leucine units have an estimated interaction free energy of about $2.1 k_{\mathrm{B}} T$ from simulation, which compares well to the experimental range 3.0-3.4 $k_{\mathrm{B}} T$, and both suggest that hydrophobic amino acids interact independently and additively with the surface. Our results demonstrate how single-molecule measurements with AFM can complement and validate theoretical data at solid/liquid interfaces, providing a roadmap to increase precision. In turn, modeling of the pulling process shows how an individual surface-to-molecule interaction dynamically breaks, and how equilibrium interaction free energies can be estimated from such nonequilibrium experiments in both simulation and experiment. Our approach will prove useful for designing synergistic experimental and simulation studies for understanding molecular thermodynamics and fluctuations at solid/liquid interfaces under dynamic load in a wide range of fields, including interactions at biointerfaces and adhesive interactions in technological systems in a broad context ranging from, e.g., cell-surface, cell-cell interactions to adhesive bonding in biomedical applications.

\section{MATERIALS AND METHODS}

Chemicals and Materials. Highest available purity 1-undecanethiol (UDT, 98\%), 11-mercaptoundecanol, 16-mercaptohexadecanoic acid, 11-thiol-undecanoic acid, N-hydroxysuccimid (NHS), 1-ethyl-3(3-dimethylaminopropyl)-carbodiimide hydrochloride (EDC $\cdot \mathrm{HCl}$ ), phosphate buffered saline (PBS), ethylenediamine, and 0,0'-bis(2carboxyethyl)dodecaethyleneglycole and all solvents were used as received (Sigma Aldrich). Peptide sequences were purchased from jpt Peptide Technologies GmbH (Berlin, Germany). Milli-Q water (Millipore, resistivity of $\geq 18 \mu \Omega$-cm, TOC below $2 \mathrm{ppb}$ ) is used for preparing $\mathrm{NaCl}$ solutions $\left(\mathrm{pH}=5.5-5.7, c_{\mathrm{NaCl}}=1 \mathrm{mmol}\right.$ for all measurements).

AFM Measurements. All AFM measurements are performed in a home-built closed fluid cell (Nanowizard 1, JPK Instruments, Germany) using gold-coated silicon tips (CONT-GB, BudgetSensors).
The thermal noise method is used to estimate the spring constants of different cantilevers, ${ }^{62}$ typically varying from 250 to $500 \mathrm{pN} / \mathrm{nm}$. Deflection versus displacement raw data are converted into force versus relative distance profiles using the JPK software. The closest approach is set to $D=0$. At least 2000 force runs on a grid with $10 \times 10$ points (7.5 $\mathrm{nm}$ spacing) are measured for various approach rates (0.1, 0.5, 1, $1.5,2 \mu \mathrm{m} / \mathrm{s}$ ), which is also known as force mapping mode. A maximum force of $500 \mathrm{pN}$ was set during contact with the sample, in order to avoid blunting of the rather soft gold tips. During contact, no dwell time is set, i.e., the tip is retracted immediately at every position during each force run. All data are recorded and analyzed using JPK data processing software. Force profiles showing specific singlemolecule events are specifically analyzed, and both rupture forces as well as the work of each trajectory are extracted from the data as described below.

AFM Tip Preparation. Gold-coated silicon tips were cleaned in $\mathrm{H}_{2} \mathrm{SO}_{4}, \mathrm{H}_{2} \mathrm{O}, \mathrm{H}_{2} \mathrm{O}$, and EtOH (each step $1 \mathrm{~min}$ ), dried in $\mathrm{N}_{2}$ stream, and placed in a $1 \mathrm{mM}$ ethanolic solution of 1-undecanethiol. After 18$24 \mathrm{~h}$, the tips were taken out of the solution, washed with hexane and ethanol to remove any unbound thiols, and dried in a $\mathrm{N}_{2}$ stream before use for AFM experiments.

Surface Grafting. Atomically smooth gold surfaces were prepared using templating techniques described in detail elsewhere. ${ }^{22}$ Freshly stripped gold surfaces were immediately placed into a mixed SAM solution of 16-mercaptohexadecanoic acid and 11-mercaptoundecanol (ratio: 1:500). After 12-18 h, the surface was taken out of the solution, washed with hexane and ethanol to remove any unbound thiols, and dried in a $\mathrm{N}_{2}$ stream. After SAM preparation surfaces were placed in a solution of NHS (4.4 mg), EDC. HCl (18.2 mg), and 400 $\mu \mathrm{L}$ diethylamine for $2 \mathrm{~h}$ at room temperature. The surface was cleaned with PBS, $\mathrm{H}_{2} \mathrm{O}$, and $\mathrm{EtOH}$ and dried in a $\mathrm{N}_{2}$ stream. After functionalization with diamine, the surface was placed in a solution of NHS $(4.4 \mathrm{mg}), \mathrm{EDC} \cdot \mathrm{HCl}(18.2 \mathrm{mg})$ and $0,0^{\prime}$-bis(2-carboxyethyl)dodecaethyleneglycole ( $10 \mathrm{mg}$ in $10 \mathrm{~mL}$ PBS solution) for $2 \mathrm{~h}$ at room temperature. The surface was cleaned with $\mathrm{PBS}, \mathrm{H}_{2} \mathrm{O}$, and $\mathrm{EtOH}$ and dried in a $\mathrm{N}_{2}$ stream before being placed in a solution of NHS (4.4 $\mathrm{mg})$, EDC $\cdot \mathrm{HCl}(18.2 \mathrm{mg})$, and the respective peptide $(0.35 \mathrm{mmol}$ in $10 \mathrm{~mL}$ PBS solution). Note: For the peptide sequence GSGLGLGLGL, we used EtOH instead of PBS solution for the last step. Tips were taken out just before experiment, washed with PBS, $\mathrm{H}_{2} \mathrm{O}$, and $\mathrm{EtOH}$, and dried in a $\mathrm{N}_{2}$ stream. For detailed XPS analysis of the surface chemistry, see Figure S10A.

Peptide Grafting to Surfaces. Au surfaces are modified with a mixture of 11-mercaptoundecanol and 16-mercaptohexadecanoic acid providing a diluted acid group in order to further anchor model proteins by a three-step process: (1) An $\mathrm{OH} / \mathrm{COOH}$ ratio of 1:500 is used in order to prevent any lateral interactions between the grafted molecules. The slightly longer carboxylic acid enables an effective condensation eq 1 with ethylenediamine, followed by (2) a second condensation reaction with $0,0^{\prime}$-bis (2-carboxyethyl)dodecaethyleneglycole, forming the "backbone" of the protein anchor (for detailed XPS analysis of the surface chemistry see Figure S10A). The free carboxylic acid can then be (3) modified by any designed peptide sequence in a third reaction step. Table 1 shows the peptide sequences used in this work. Control experiments using surfaces after each grafting step are shown in Figure S11.

Criteria for Accepting a Single-Molecule Event. For SM-AFM analysis, the contour length is a hard selection criterion, and only profiles with $L_{\mathrm{C}} \leq 10 \mathrm{~nm}$ were evaluated. Longer molecular stretching indicates a sequence with a longer backbone (e.g., dirt). In our experiments, we observed $\ll 1 \%$ of such artificial events. The second criterion for evaluation is that the single-molecule rupture event must follow the WLC model with a contour length of $10 \mathrm{~nm}$ and a persistence length of $\sim 0.37 \mathrm{~nm}$, which indicates stretching a single polymeric chain as designed and shown in Figures 2 and S11. Only force distance profiles with specific rupture events that fulfilled the above two requirements were analyzed.

Simulated System. We constructed surfaces to emulate the properties of the experimental SAM of 11-carbon alkanethiols on an Au111 surface, as shown in Figure 8. For alkanes of this length, a rigid, 


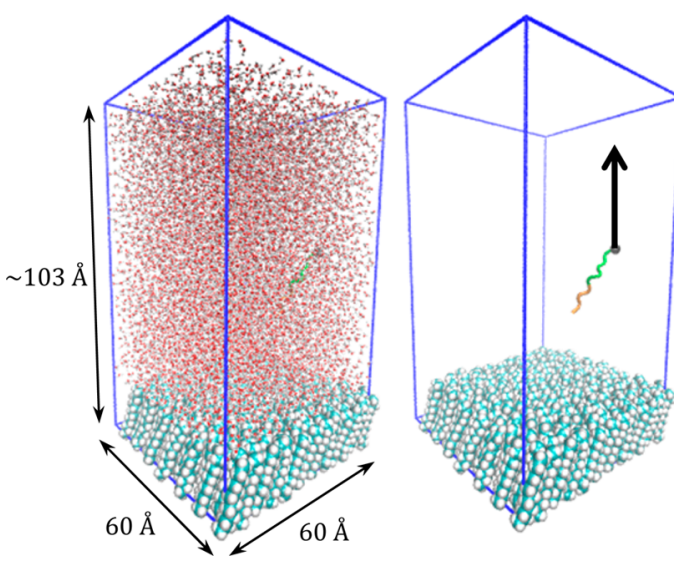

Figure 8. Dimensions of the simulation box are initially set to $60 \times$ $60 \times 100 \AA$, with pressure equilibration resulting in an increase of the box height to around $103 \AA$. The $x$ and $y$ coordinates of the system are defined to be in the plane of the surface, with the $z$ coordinate normal to the SAM. In all simulations, the peptide is pulled along only the $z$ coordinate, as shown in the right figure with waters removed.

extended structure of each chain is adopted, with a $\sqrt{3} \times \sqrt{3} \mathrm{R} 30^{\circ}$ monolayer structure. ${ }^{63,64}$ Simulated SAMs are constructed from dodecane chains aligned to this periodic structure with a nearestneighbor spacing of $4.98 \AA$. The resulting surfaces were approximately $60 \AA \times 60 \AA$, with 12 and 14 chains in the $x$ and $y$ directions, respectively. To maintain atomistic resolution and avoid introducing new parameters, atoms in dodecane chains are assigned standard AMBER force field atom types of "CT" and "H." For simplicity, charges are assigned based on the work of Siu et al. ${ }^{65}$

We minimize initial structures and then equilibrate surfaces for 200 ps with periodic boundary conditions at $300 \mathrm{~K}$ in the NVT ensemble. A position restraint (spring constant of $10 \mathrm{kcal} / \mathrm{mol} \AA^{2}$ ) fixes one end of each dodecane chain at the correct lattice location. The resulting surface structure exhibits fully extended chains with an average $\mathrm{CH}_{3}$ density at the nonfixed chain end of $21 \AA^{2} / \mathrm{CH}_{3}$ and an average chain tilt angle of around $19^{\circ}$. This tilt is smaller than the experimentally reported value of $\sim 30^{\circ}$ for chains of a similar length, ${ }^{25,28,63,64}$ even considering that the tilt angle distribution is expected to be fairly broad. $^{25,28}$ Numerous studies have tried to address simulated tilt angles in alkanethiol SAMs on Au111 surfaces, ${ }^{24,25,27,28}$ using both united atom ${ }^{24-26}$ and atomistic models, ${ }^{27,28,66}$ but very often require force-field tuning or significant atomic constraints. ${ }^{30,35}$ We allowed the chains to move freely with the assumption that the discrepancy in tilt angles is not expected to affect the qualitative predictions made in this study, as all peptides will see a similar hydrophobic surface. From the equilibration trajectory, two-dimensional radial distribution functions for the chain termini demonstrate a well-ordered structure as expected.

The simulated system lacks an explicit gold surface and thus also the $\mathrm{vdW}$ attractions that could potentially lead to lower predicted free energies. However, a rough estimate shows that long-range vdW attractions cause the slope in Figure 3 to increase by $<0.1 k_{\mathrm{B}} T$ /leucine, as shown in the SI.

A periodic box is defined with dimensions of $\sim 60 \times 60 \times 100 \AA^{3}$ (Figure 8), which accommodates an extended peptide length of $\sim 34 \AA$ and a nonbonded cutoff of $8 \AA$. . 10,000 waters are added via the crystal solvation modules in AMBER. Solvated systems are energy minimized and then equilibrated with two separate MD simulations: first heating from 100 to $300 \mathrm{~K}$ over 500 ps at constant volume, then $1 \mathrm{~ns}$ of NPT dynamics at 1 bar.

Simulation Protocol. MD in all steps are carried out using PMEMD in the AMBER 14 package with explicit TIP3P waters ${ }^{67}$ and the ff14SB force field. ${ }^{68}$ Though past studies have shown that the choice of water model can influence the precise behavior of hydrophobic phenomena, ${ }^{69,70}$ here we maintain the TIP3P model to ensure consistency with the chosen protein force field. Throughout, a
2 fs time step is employed, and SHAKE and SETTLE are used to constrain all bonds involving hydrogen atoms. ${ }^{71,72}$ We use a Langevin thermostat with a collision frequency of $2 \mathrm{ps}^{-1}$ and an anisotropic Berendsen barostat. ${ }^{73}$ Both are weakly coupled, and we verify the absence of significant density variations during nonequilibrium pulling.

In nonequilibrium simulations, the pulling coordinate is defined as the height of the pulled peptide end (the $\alpha$-carbon opposite the hydrophobic leucine residues) above the fixed carbons of the SAM (Figure 1). Pulling is performed at rates of $10 \AA / \mathrm{ns}$ and $2 \AA / \mathrm{ns}$ with a spring constant of $0.251 \mathrm{kcal} / \mathrm{mol} \cdot \AA^{2}(350 \mathrm{pN} / \mathrm{nm})$, using a custom restraint potential in AMBER that pulls only in the $z$ direction, normal to the surface. To generate an ensemble of initial conformations for forward pulling runs, we simulate the entire system for $20 \mathrm{~ns}$ at $300 \mathrm{~K}$ and $1 \mathrm{bar}$ using a flat-bottom harmonic restraint with spring constant $0.8 \mathrm{kcal} / \mathrm{mol} \cdot \AA^{2}$ to keep the peptide end within $18 \AA$ above the bottom of the surface. A total of 30 configurations for fast-pulling runs, or 60 for slow-pulling, evenly spaced in time, are chosen from the last $18 \mathrm{~ns}$ of this trajectory as seeds for forward pulling runs, with randomly assigned initial velocities at $300 \mathrm{~K}$.

Initial conformations for the reverse runs (toward the surface) are obtained from bulk simulations. Each peptide is solvated in a water box that extends at least $20 \AA$ on each side, then simulated for 100 ns at $300 \mathrm{~K}$ and 1 bar. Thirty (for fast-pulling) or 60 (for slow-pulling) peptide conformations from the last $90 \mathrm{~ns}$ are selected at even time intervals to be placed $60 \AA$ away from the position of the fixed carbon atoms in the surface. Before performing reverse pulling runs, the resulting system is solvated and equilibrated as already described, except that the $\alpha$-carbon positions of the peptide are restrained during heating and density equilibration to preserve the chosen configuration.

In equilibrium umbrella-sampling simulations, the setup is similar to that described above for equilibrating the peptide on the surface. Instead of restraining the pulled $\alpha$ carbon, we instead restrain the center of mass of all leucine residues, or the center of mass of the isobutane molecule, using a harmonic potential with a spring constant of $2.0 \mathrm{kcal} / \mathrm{mol} \cdot \AA^{2}$. Depending on the peptide, $23-28$ windows from 18.0 to $50.0 \AA$ away from the fixed carbon atoms of the SAM are used (17 windows 16.0-29.0 $\AA$ away for isobutane). For each window, 50 ns (10 ns for isobutane) of simulation time is generated and subsequently used to compute a PMF, except for $(\mathrm{GS})_{4} \mathrm{GL}$, for which $80 \mathrm{~ns}$ was used. This is necessary to converge the calculations due to large conformational fluctuations in the unrestrained glycine/serine portion of the peptide.

Free Energy Estimates. With the exception of free energies reported from umbrella sampling, all estimates of the free energy are computed with $\mathrm{JE}^{39}$ using only forward realizations of the nonequilibrium pulling process. Variances in these free energy changes are estimated using 10,000 bootstrap resamples of the original data. To analyze umbrella sampling simulations, the free energy was computed as the difference between the invariant, asymptotic portion at far distances, and the minimum of PMFs generated using the MBAR algorithm ${ }^{74}$ implemented in pymbar. ${ }^{75}$ It should be noted that these free energy differences are subtly different than those computed via JE in two ways. First, they represent the free energy for moving the center of mass of leucine residues to a position far from the surface rather than the $\alpha$ carbon opposite these hydrophobic residues. Second, they do not include the free energy of applying a harmonic restraint, as the effect of this has been removed from the PMF through MBAR. To address the first, PMFs computed over both leucine center of mass and pulled $\alpha$ carbon indicate that this free energy difference is similar (Figure S9), though significantly more complicated for the case of the $\alpha$ carbon. In the case of the second difference, this contribution is expected to be small, as mentioned earlier. ${ }^{36}$

With simulations, it is also possible in principle to make use of reverse pulling simulations of the peptide toward the surface. Reverse moves tend to improve convergence of free energy estimates ${ }^{76-78}$ and allow use of the $\mathrm{BAR}^{79-81}$ approach, which often improves upon the forward-only JE for an equivalent number of trajectories. ${ }^{52,78}$ While this proves true for the simulations at faster pulling rates, poor convergence of the reverse distributions in slower pulling simulations proves detrimental to these estimates. Comparisons of free energy 
estimates from both JE and BAR, as well as detailed methodologies to account for statistical and sampling errors in these estimators, are discussed in the SI.

\section{ASSOCIATED CONTENT}

\section{S Supporting Information}

The Supporting Information is available free of charge on the ACS Publications website at DOI: 10.1021/acsnano.6b06360.

Detailed discussion of bias in free energy estimation techniques, extended analysis of simulation results, experimental validation data, and extended experimental data sets (PDF)

Detachment for $(\mathrm{GS})_{3} \mathrm{~L}_{4}(\mathrm{MPG})$

\section{AUTHOR INFORMATION}

\section{Corresponding Authors}

*E-mail: shell@engineering.ucsb.edu.

*E-mail: Markus.Valtiner@chemie.tu-freiberg.de.

\section{ORCID}

Markus Valtiner: 0000-0001-5410-1067

\section{Author Contributions}

"These authors contributed equally. M.V. and P.S. designed the experiments. P.S. and T.U. conducted the experiments and analyzed the results. M.S.S., J.I.M., and D.J.S. designed, analyzed, and discussed all of the simulation results. J.I.M. performed and analyzed the nonequilibrium simulations. D.J.S. conducted implicit solvent simulations and assisted in the interpretation of all simulation results.

\section{Notes}

The authors declare no competing financial interest.

\section{ACKNOWLEDGMENTS}

We gratefully acknowledge the NSF (project DMR-1312548) and the German Research Foundation DFG (project no. VA 689/3-1) for financial support in the framework of the NSF/ DFG scheme Materials World Network: Fundamentals of Peptide Materials - Experimental and Simulation Probes. We also thank the Center for Scientific Computing at UCSB (NSF grant CNS-0960316) and the HYDRA system at Max Planck Computing and Data Facility for generously providing computing resources. We are particularly indebted to Paul Weakliem for assistance and advice in utilizing GPUs. T.U. acknowledges funding through the International Max Planck Research School (IMPRS) SURMAT. J.I.M. acknowledges support from the National Science Foundation Graduate Research Fellowship Program under grant no. DGE 1144085. M.V. acknowledges support by the European Research Council (Grant no. 677663, from 07/2016).

\section{REFERENCES}

(1) Ducker, W. A.; Mastropietro, D. Forces between Extended Hydrophobic Solids: Is There a Long-Range Hydrophobic Force? Curr. Opin. Colloid Interface Sci. 2016, 22, 51-58.

(2) Meagher, L.; Craig, V. S. J. Effect of Dissolved Gas and Salt on the Hydrophobic Force between Polypropylene Surfaces. Langmuir 1994, 10, 2736-2742.

(3) Donaldson, S. H.; Røyne, A.; Kristiansen, K.; Rapp, M. V.; Das, S.; Gebbie, M. A.; Lee, D. W.; Stock, P.; Valtiner, M.; Israelachvili, J. Developing a General Interaction Potential for Hydrophobic and Hydrophilic Interactions. Langmuir 2015, 31, 2051-2064.

(4) Mastropietro, D. J.; Ducker, W. A. Forces between Hydrophobic Solids in Concentrated Aqueous Salt Solution. Phys. Rev. Lett. 2012, 108, 106101.
(5) Stock, P.; Utzig, T.; Valtiner, M. Direct and Quantitative AFM Measurements of the Concentration and Temperature Dependence of the Hydrophobic Force Law at Nanoscopic Contacts. J. Colloid Interface Sci. 2015, 446, 244-251.

(6) Tabor, R. F.; Wu, C.; Grieser, F.; Dagastine, R. R.; Chan, D. Y. C. Measurement of the Hydrophobic Force in a Soft Matter System. J. Phys. Chem. Lett. 2013, 4, 3872-3877.

(7) Scheu, R.; Rankin, B. M.; Chen, Y.; Jena, K. C.; Ben-Amotz, D.; Roke, S. Charge Asymmetry at Aqueous Hydrophobic Interfaces and Hydration Shells. Angew. Chem., Int. Ed. 2014, 53, 9560-9563.

(8) Scheu, R.; Chen, Y.; Subinya, M.; Roke, S. Stern Layer Formation Induced by Hydrophobic Interactions: A Molecular Level Study. J. Am. Chem. Soc. 2013, 135, 19330-19335.

(9) Mezger, M.; Sedlmeier, F.; Horinek, D.; Reichert, H.; Pontoni, D.; Dosch, H. On the Origin of the Hydrophobic Water Gap: An XRay Reflectivity and MD Simulation Study. J. Am. Chem. Soc. 2010, 132, 6735-6741.

(10) Ben-Amotz, D. Water-Mediated Hydrophobic Interactions. Annu. Rev. Phys. Chem. 2016, 67, 617-638.

(11) Hillyer, M. B.; Gibb, B. C. Molecular Shape and the Hydrophobic Effect. Annu. Rev. Phys. Chem. 2016, 67, 307-329.

(12) Dill, K. A.; Ozkan, S. B.; Shell, M. S.; Weikl, T. R. The Protein Folding Problem. Annu. Rev. Biophys. 2008, 37, 289-316.

(13) Fairman, R.; Akerfeldt, K. Peptides as Novel Smart Materials. Curr. Opin. Struct. Biol. 2005, 15, 453-463.

(14) Grove, T. Z.; Regan, L. New Materials from Proteins and Peptides. Curr. Opin. Struct. Biol. 2012, 22, 451-456.

(15) Krysiak, S.; Liese, S.; Netz, R. R.; Hugel, T. Peptide Desorption Kinetics from Single Molecule Force Spectroscopy Studies. J. Am. Chem. Soc. 2014, 136, 688-697.

(16) Horinek, D.; Serr, A.; Geisler, M.; Pirzer, T.; Slotta, U.; Lud, S. Q.; Garrido, J. A.; Scheibel, T.; Hugel, T.; Netz, R. R. Peptide Adsorption on a Hydrophobic Surface Results from an Interplay of Solvation, Surface, and Intrapeptide Forces. Proc. Natl. Acad. Sci. U. S. A. 2008, 105, 2842-2847.

(17) Ma, C. D.; Wang, C.; Acevedo-Velez, C.; Gellman, S. H.; Abbott, N. L. Modulation of Hydrophobic Interactions by Proximally Immobilized Ions. Nature 2015, 517, 347-350.

(18) Tych, K. M.; Hughes, M. L.; Bourke, J.; Taniguchi, Y.; Kawakami, M.; Brockwell, D. J.; Dougan, L. Optimizing the Calculation of Energy Landscape Parameters from Single-Molecule Protein Unfolding Experiments. Phys. Rev. E - Stat. Nonlinear, Soft Matter Phys. 2015, 91, 012710.

(19) Schwierz, N.; Horinek, D.; Liese, S.; Pirzer, T.; Balzer, B. N.; Hugel, T.; Netz, R. R. On the Relationship between Peptide Adsorption Resistance and Surface Contact Angle: A Combined Experimental and Simulation Single-Molecule Study. J. Am. Chem. Soc. 2012, 134, 19628-19638.

(20) Thyparambil, A. A.; Wei, Y.; Latour, R. A. Determination of Peptide - Surface Adsorption Free Energy for Material Surfaces Not Conducive to SPR or QCM Using AFM. Langmuir 2012, 28, 56875694.

(21) Ray, C.; Gu, C.; Brown, J. R.; Kirkpatrick, A.; Akhremitchev, B. B. Anisotropy of Pairwise Interactions between Hexadecanes in Water Measured by AFM Force Spectroscopy. J. Phys. Chem. C 2008, 112, 18164-18172.

(22) Hegner, M.; Wagner, P.; Semenza, G. Ultralarge Atomically Flat Template-Stripped Au Surfaces for Scanning Probe Microscopy. Surf. Sci. 1993, 291, 39-46.

(23) Hinterdorfer, P.; Dufrene, Y. F. Detection and Localization of Single Molecular Recognition Events Using Atomic Force Microscopy. Nat. Methods 2006, 3, 347-355.

(24) Rai, B.; Sathish, P.; Malhotra, C. P.; Pradip; Ayappa, K. G. Molecular Dynamic Simulations of Self-Assembled Alkylthiolate Monolayers on an $\mathrm{Au}(\mathrm{III})$ Surface. Langmuir 2004, 20, 3138-3144.

(25) Ramin, L.; Jabbarzadeh, A. Odd-Even Effects on the Structure, Stability, and Phase Transition of Alkanethiol Self-Assembled Monolayers. Langmuir 2011, 27, 9748-9759. 
(26) Srivastava, P.; Chapman, W. G.; Laibinis, P. E. Molecular Dynamics Simulation of Oxygen Transport through N-Alkanethiolate Self-Assembled Monolayers on Gold and Copper. J. Phys. Chem. B 2009, 113, 456-464.

(27) Vemparala, S.; Karki, B. B.; Kalia, R. K.; Nakano, A.; Vashishta, P. Large-Scale Molecular Dynamics Simulations of Alkanethiol SelfAssembled Monolayers. J. Chem. Phys. 2004, 121, 4323.

(28) Wang, Y.; Solano Canchaya, J. G.; Dong, W.; Alcamí, M.; Busnengo, H. F.; Martín, F. Chain-Length and Temperature Dependence of Self-Assembled Monolayers of Alkylthiolates on $\mathrm{Au}(111)$ and $\mathrm{Ag}(111)$ Surfaces. J. Phys. Chem. A 2014, 118, 41384146.

(29) Collier, G.; Vellore, N. A.; Yancey, J. A.; Stuart, S. J.; Latour, R. A. Comparison between Empirical Protein Force Fields for the Simulation of the Adsorption Behavior of Structured LK Peptides on Functionalized Surfaces. Biointerphases 2012, 7, 24-43.

(30) Deighan, M.; Pfaendtner, J. Exhaustively Sampling Peptide Adsorption with Metadynamics. Langmuir 2013, 29, 7999-8009.

(31) Mijajlovic, M.; Penna, M. J.; Biggs, M. J. Free Energy of Adsorption for a Peptide at a Liquid/solid Interface via Nonequilibrium Molecular Dynamics. Langmuir 2013, 29, 2919-2926.

(32) Shen, J.-W.; Wu, T.; Wang, Q.; Pan, H.-H. Molecular Simulation of Protein Adsorption and Desorption on Hydroxyapatite Surfaces. Biomaterials 2008, 29, 513-532.

(33) Soliman, W.; Bhattacharjee, S.; Kaur, K. Adsorption of an Antimicrobial Peptide on Self-Assembled Monolayers by Molecular Dynamics Simulation. J. Phys. Chem. B 2010, 114, 11292-11302.

(34) Abramyan, T.; Collier, G.; Kucukkal, T. G.; Li, X.; Snyder, J. A.; Thyparambil, A. A.; Vellore, N. A.; Wei, Y.; Yancey, J. A.; Stuart, S. J.; et al. Understanding Protein-Surface Interactions at the Atomistic Level through the Synergistic Development of Experimental and Molecular Simulation Methods. ACS Symp. Ser. 2012, 1120, 197-228.

(35) Raut, V. P.; Agashe, M. A.; Stuart, S. J.; Latour, R. A. Molecular Dynamics Simulations of Peptide - Surface Interactions. Langmuir 2005, 21, 1629-1639.

(36) Raman, S.; Utzig, T.; Baimpos, T.; Ratna Shrestha, B.; Valtiner, M. Deciphering the Scaling of Single-Molecule Interactions Using Jarzynski's Equality. Nat. Commun. 2014, 5, 5539.

(37) Pashley, R. M.; Israelachvili, J. N. DLVO and Hydration Forces between Mica Surfaces in $\mathrm{Mg} 2+, \mathrm{Ca} 2+, \mathrm{Sr} 2+$, and $\mathrm{Ba} 2+$ Chloride Solutions. J. Colloid Interface Sci. 1984, 97, 446-455.

(38) Valtiner, M.; Kristiansen, K.; Greene, G. W.; Israelachvili, J. N. Effect of Surface Roughness and Electrostatic Surface Potentials on Forces between Dissimilar Surfaces in Aqueous Solution. Adv. Mater. 2011, 23, 2294-2299.

(39) Jarzynski, C. Nonequilibrium Equality for Free Energy Differences. Phys. Rev. Lett. 1997, 78, 2690-2693.

(40) Utzig, T.; Raman, S.; Valtiner, M. Scaling from Single Molecule to Macroscopic Adhesion at Polymer/metal Interfaces. Langmuir 2015, 31, 2722-2729.

(41) Stock, P.; Utzig, T.; Valtiner, M. Soft Matter Interactions at the Molecular Scale: Interaction Forces and Energies between Single Hydrophobic Model Peptides. Phys. Chem. Chem. Phys. 2017, 19, $4216-4221$.

(42) Kienberger, F.; Pastushenko, V. P.; Kada, G.; Gruber, H. J.; Riener, C.; Schindler, H.; Hinterdorfer, P. Static and Dynamical Properties of Single Poly(Ethylene Glycol) Molecules Investigated by Force Spectroscopy. Single Mol. 2000, 1, 123-128.

(43) Marko, J. F.; Siggia, E. D. Stretching DNA. Macromolecules 1995, 28, 8759-8770.

(44) Hummer, G.; Szabo, A. Free Energy Reconstruction from Nonequilibrium Single-Molecule Pulling Experiments. Proc. Natl. Acad. Sci. U. S. A. 2001, 98, 3658-3661.

(45) Hummer, G.; Szabo, A. Free Energy Surfaces from SingleMolecule Force Spectroscopy. Acc. Chem. Res. 2005, 38, 504-513.

(46) Israelachvili, J. N. Intermolecular and Surface Forces; 3rd ed.; Elsevier: Burlington, MA, 2011.
(47) Smith, D. E.; Zhang, L.; Haymet, A. D. J. Entropy of Association of Methane in Water: A New Molecular Dynamics Computer Simulation. J. Am. Chem. Soc. 1992, 114, 5875-5876.

(48) Ben-Naim, A.; Wilf, J.; Yaacobi, M. Hydrophobic Interactions in Light and Heavy Water. J. Phys. Chem. 1973, 77, 95-102.

(49) Tucker, E. E.; Lane, E. H.; Christian, S. D. Vapor Pressure Studies of Hydrophobic Interactions. Formation of Benzene-Benzene and Cyclohexane-Cyclohexanol Dimers in Dilute Aqueous Solution. J. Solution Chem. 1981, 10, 1-20.

(50) Li, I. T. S.; Walker, G. C. Single Polymer Studies of Hydrophobic Hydration. Acc. Chem. Res. 2012, 45, 2011-2021.

(51) Gore, J.; Ritort, F.; Bustamante, C. Bias and Error in Estimates of Equilibrium Free-Energy Differences from Nonequilibrium Measurements. Proc. Natl. Acad. Sci. U. S. A. 2003, 100, 12564-12569.

(52) Shirts, M. R.; Pande, V. S. Comparison of Efficiency and Bias of Free Energies Computed by Exponential Averaging, the Bennett Acceptance Ratio, and Thermodynamic Integration. J. Chem. Phys. 2005, 122, 144107 .

(53) Chandler, D. Interfaces and the Driving Force of Hydrophobic Assembly. Nature 2005, 437, 640-647.

(54) Acharya, H.; Vembanur, S.; Jamadagni, S. N.; Garde, S. Mapping Hydrophobicity at the Nanoscale: Applications to Heterogeneous Surfaces and Proteins. Faraday Discuss. 2010, 146, 353-365.

(55) Remsing, R. C.; Weeks, J. D. Hydrophobicity Scaling of Aqueous Interfaces by an Electrostatic Mapping. J. Phys. Chem. B 2015, $119,9268$.

(56) Chaimovich, A.; Shell, M. S. Tetrahedrality and Structural Order for Hydrophobic Interactions in a Coarse-Grained Water Model. Phys. Rev. E 2014, 89, 22140.

(57) Bernal, J. D.; Fowler, R. H. A Theory of Water and Ionic Solution, with Particular Reference to Hydrogen and Hydroxyl Ions. J. Chem. Phys. 1933, 1, 515-548.

(58) Chau, P. L.; Hardwick, A. J. A New Order Parameter for Tetrahedral Configurations. Mol. Phys. 1998, 93, 511-518.

(59) Errington, J. R.; Debenedetti, P. G. Relationship between Structural Order and the Anomalies of Liquid Water. Nature 2001, 409, 318-321.

(60) Clark, G. N. I.; Cappa, C. D.; Smith, J. D.; Saykally, R. J.; HeadGordon, T. The Structure of Ambient Water. Mol. Phys. 2010, 108, 1415-1433.

(61) Ferguson, A. L.; Debenedetti, P. G.; Panagiotopoulos, A. Z. Solubility and Molecular Conformations of N -Alkane Chains in Water. J. Phys. Chem. B 2009, 113, 6405-6414.

(62) Burnham, N. A.; Chen, X.; Hodges, C. S.; Matei, G. A.; Thoreson, E. J.; Roberts, C. J.; Davies, M. C.; Tendler, S. J. B. Comparison of Calibration Methods for Atomic-Force Microscopy Cantilevers. Nanotechnology 2003, 14, 1-6.

(63) Vericat, C.; Vela, M. E.; Benitez, G. A.; Gago, J. A. M.; Torrelles, X.; Salvarezza, R. C. Surface Characterization of Sulfur and Alkanethiol Self-Assembled Monolayers on $\mathrm{Au}(111)$. J. Phys.: Condens. Matter 2006, 18, R867-R900.

(64) Vericat, C.; Vela, M. E.; Benitez, G.; Carro, P.; Salvarezza, R. C. Self-Assembled Monolayers of Thiols and Dithiols on Gold: New Challenges for a Well-Known System. Chem. Soc. Rev. 2010, 39, 1805.

(65) Siu, S. W. I.; Pluhackova, K.; Böckmann, R. A. Optimization of the OPLS-AA Force Field for Long Hydrocarbons. J. Chem. Theory Comput. 2012, 8, 1459-1470.

(66) Canchaya, J. G. S.; Wang, Y.; Alcamí, M.; Martín, F.; Busnengo, H. F. Study of the Interaction between Short Alkanethiols from $\mathrm{Ab}$ Initio Calculations. Phys. Chem. Chem. Phys. 2010, 12, 7555.

(67) Jorgensen, W. L.; Chandrasekhar, J.; Madura, J. D.; Impey, R. W.; Klein, M. L. Comparison of Simple Potential Functions for Simulating Liquid Water. J. Chem. Phys. 1983, 79, 926.

(68) Maier, J. A.; Martinez, C.; Kasavajhala, K.; Wickstrom, L.; Hauser, K. E.; Simmerling, C. ff14SB: Improving the Accuracy of Protein Side Chain and Backbone Parameters from ff99SB. J. Chem. Theory Comput. 2015, 11, 3696-3713. 
(69) Paschek, D. Temperature Dependence of the Hydrophobic Hydration and Interaction of Simple Solutes: An Examination of Five Popular Water Models. J. Chem. Phys. 2004, 120, 6674-6690.

(70) Ashbaugh, H. S.; Collett, N. J.; Hatch, H. W.; Staton, J. A. Assessing the Thermodynamic Signatures of Hydrophobic Hydration for Several Common Water Models. J. Chem. Phys. 2010, 132, 124504.

(71) Ryckaert, J.-P.; Ciccotti, G.; Berendsen, H. J. C. Numerical Integration of the Cartesian Equations of Motion of a System with Constraints: Molecular Dynamics of N-Alkanes. J. Comput. Phys. 1977, 23, 327-341.

(72) Miyamoto, S.; Kollman, P. A. Settle: An Analytical Version of the SHAKE and RATTLE Algorithm for Rigid Water Models. J. Comput. Chem. 1992, 13, 952-962.

(73) Berendsen, H. J. C.; Postma, J. P. M.; van Gunsteren, W. F.; DiNola, A.; Haak, J. R. Molecular Dynamics with Coupling to an External Bath. J. Chem. Phys. 1984, 81, 3684.

(74) Shirts, M. R.; Chodera, J. D. Statistically Optimal Analysis of Samples from Multiple Equilibrium States. J. Chem. Phys. 2008, 129, 124105.

(75) Chodera, J. D.; Shirts, M. R. A python implementation of the multistate Bennet acceptance ratio (MBAR); version 3.0.0beta2 https:// simtk.org/home/pymbar.

(76) Kosztin, I.; Barz, B.; Janosi, L. Calculating Potentials of Mean Force and Diffusion Coefficients from Nonequilibrium Processes without Jarzynski's Equality. J. Chem. Phys. 2006, 124, 064106.

(77) Nategholeslam, M.; Gray, C. G.; Tomberli, B. Implementation of the Forward-Reverse Method for Calculating the Potential of Mean Force Using a Dynamic Restraining Protocol. J. Phys. Chem. B 2014, $118,14203-14214$.

(78) Pohorille, A.; Jarzynski, C.; Chipot, C. Good Practices in FreeEnergy Calculations. J. Phys. Chem. B 2010, 114, 10235-10253.

(79) Bennett, C. H. Efficient Estimation of Free Energy Differences from Monte Carlo Data. J. Comput. Phys. 1976, 22, 245-268.

(80) Crooks, G. Path-Ensemble Averages in Systems Driven far from Equilibrium. Phys. Rev. E: Stat. Phys., Plasmas, Fluids, Relat. Interdiscip. Top. 2000, 61, 2361-2366.

(81) Shirts, M. R.; Bair, E.; Hooker, G.; Pande, V. S. Equilibrium Free Energies from Nonequilibrium Measurements Using MaximumLikelihood Methods. Phys. Rev. Lett. 2003, 91, 140601. 